\title{
Echocardiography in combined discrete and hypertrophic subaortic stenosis
}

\author{
RICHARD CHARLES, CAROLYN MAKIN, NORMAN COULSHED, \\ AND DAVID HAMILTON \\ From the Regional Cardiac Centre, Sefton General Hospital, Liverpool
}

\begin{abstract}
A 10-year-old boy with discrete subaortic stenosis had coexisting abnormal systolic anterior motion of the mitral valve, demonstrated by echocardiography, a sign normally taken as indicating the presence of idiopathic hypertrophic subaortic stenosis. Surgical removal of a fibromuscular diaphragm abolished the echocardiographic signs of discrete subaortic stenosis but abnormal systolic anterior motion of the mitral valve persisted. A severe low cardiac output state complicated immediate recovery after removal of the left ventricle outflow obstruction, and was overcome only with considerable difficulty. The presence of hypertrophied septal muscle, and the associated small left ventricular cavity size, was thought to be the immediate cause of these problems, so that recognition of marked septal hypertrophy, together with abnormal anterior systolic movement of the mitral valve, should serve as a warning that similar difficulties are likely to be encountered by other patients, after removal of the obstruction in subaortic stenosis. In our experience other forms of left ventricle outflow tract obstruction have not been found to show such a marked degree of asymmetric septal hypertrophy, but this does not mean it may not occur.
\end{abstract}

Discrete subaortic stenosis (DSAS) is a relatively frequent cause of left ventricular outflow tract (LVOT) obstruction, the morphological subtypes of which have unique operative and prognostic characteristics. ${ }^{1}$ In type 1 a thin, discrete membrane obstructs outflow but is not associated with narrowing of the tract. In type 2 a thicker fibromuscular ring is often associated with muscular hypertrophy which narrows the tract, and encroaches on the anterior cusp of the mitral valve. ${ }^{2}$ These structures may be difficult to delineate even at cardiac catheterisation.

Recently, echocardiography has been used to identify the condition, ${ }^{34}$ and to distinguish it from idiopathic hypertrophic subaortic stenosis. ${ }^{3}$ Some patients, however, show features of both discrete and diffuse outflow tract obstruction, the more diffuse types being the least amenable to surgery and giving the poorest post-operative results. ${ }^{2}$ Clear echocardiographic evidence for two coexisting mechanisms of LVOT obstruction was demonstrated in the present case. The report

Address for reprint requests: Dr N Coulshed, Regional Cardiac Centre, Sefton General Hospital, Smithdown Road, Liverpool L15 2HE. emphasises that careful echocardiographic assessment may, in addition to establishing a diagnosis of DSAS, allow anticipation of serious operative complications, and possibly give information about the long-term prognosis.

\section{Case report}

A 10-year-old boy was referred for investigation of a systolic murmur. A heart murmur was noted at birth and again at age 5 years when the ECG and chest radiograph were normal. By age 7 years the voltage criteria for left ventricular hypertrophy were present, but he remained active and asymptomatic; in particular there was no chest pain or syncope. There was no family history of heart disease.

On examination he was $136 \mathrm{~cm}$ tall and weighed $26.5 \mathrm{~kg}$. There was no cyanosis. The pulse was $80 / \mathrm{min}$, regular, and small volume. Blood pressure was $100 / 70 \mathrm{mmHg}$. The apex beat was thrusting and displaced laterally. A systolic thrill was palpable over the whole precordium and neck. Harsh midsystolic and soft early diastolic murmurs were heard maximally in the aortic 
area and left sternal edge respectively. There was no ejection sound.

The ECG showed gross left ventricular hypertrophy with $\mathrm{T}$ wave inversion in leads $\mathrm{I}$, AV1 and V5-6. A chest radiograph confirmed left ventricular enlargement and dilatation of the aorta. Cardiac catheterisation revealed a systolic gradient of $100 \mathrm{mmHg}$ across the left ventricular outflow tract (LVOT) and left ventricular angiography demonstrated a ring obstruction below the aortic leailets. At operation, marked hypertrophy of the left ventricle was noted. There were three mildly thickened aortic valve cusps immediately beneath which was a white fibromuscular diaphragm particularly well formed posteriorly and attached to the anterior mitral leaflet in this area. Anteriorly and to the left there was a fibrous ledge which widened into a muscular base beneath the commissure between the right and left coronary cusps. The orifice of this obstruction was $6 \mathrm{~mm}$ in diameter. The obstruction was widely resected, dissecting the fibrous bar down to the mitral leaflet, so that a $15 \mathrm{~mm}$ dilator passed freely into the left ventricular cavity. Initially the circulation could not adequately be re-established and manual

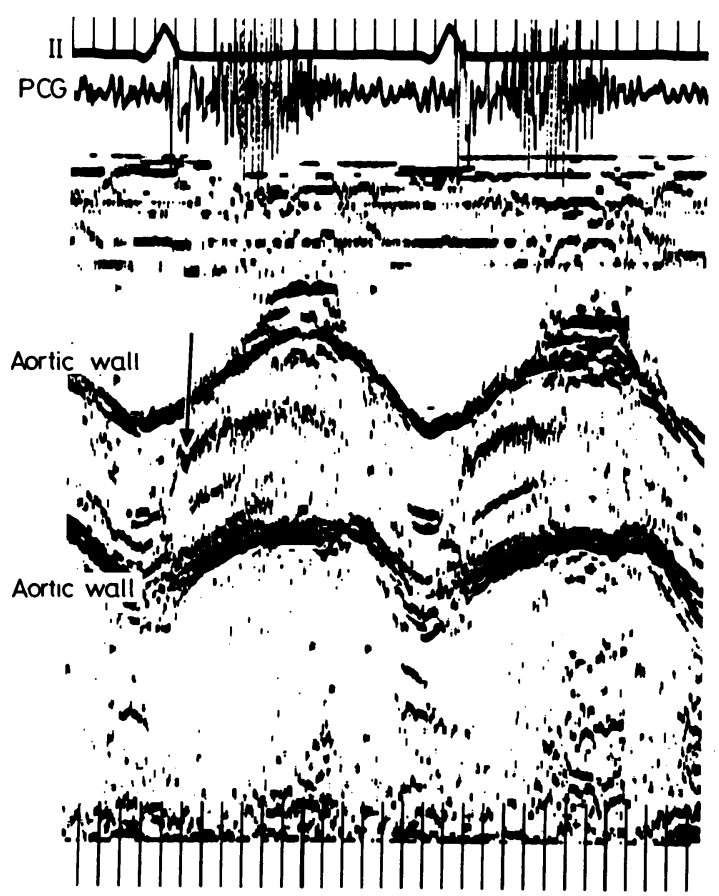

Fig 1 Echocardiogram of aortic valve $(100 \mathrm{~mm} / \mathrm{s})$ showing early systolic partial closure (arrowed) followed by prominent fluttering of the valve cusps. massage was required. Partial bypass was recommenced and continued for two and three-quarter hours until the left ventricle was able gradually to maintain perfusion. Thereafter postoperative progress was satisfactory.

Echocardiography was particularly informative. Before operation the aortic valve cusps showed a sharp early systolic partial closure (arrowed) fol-

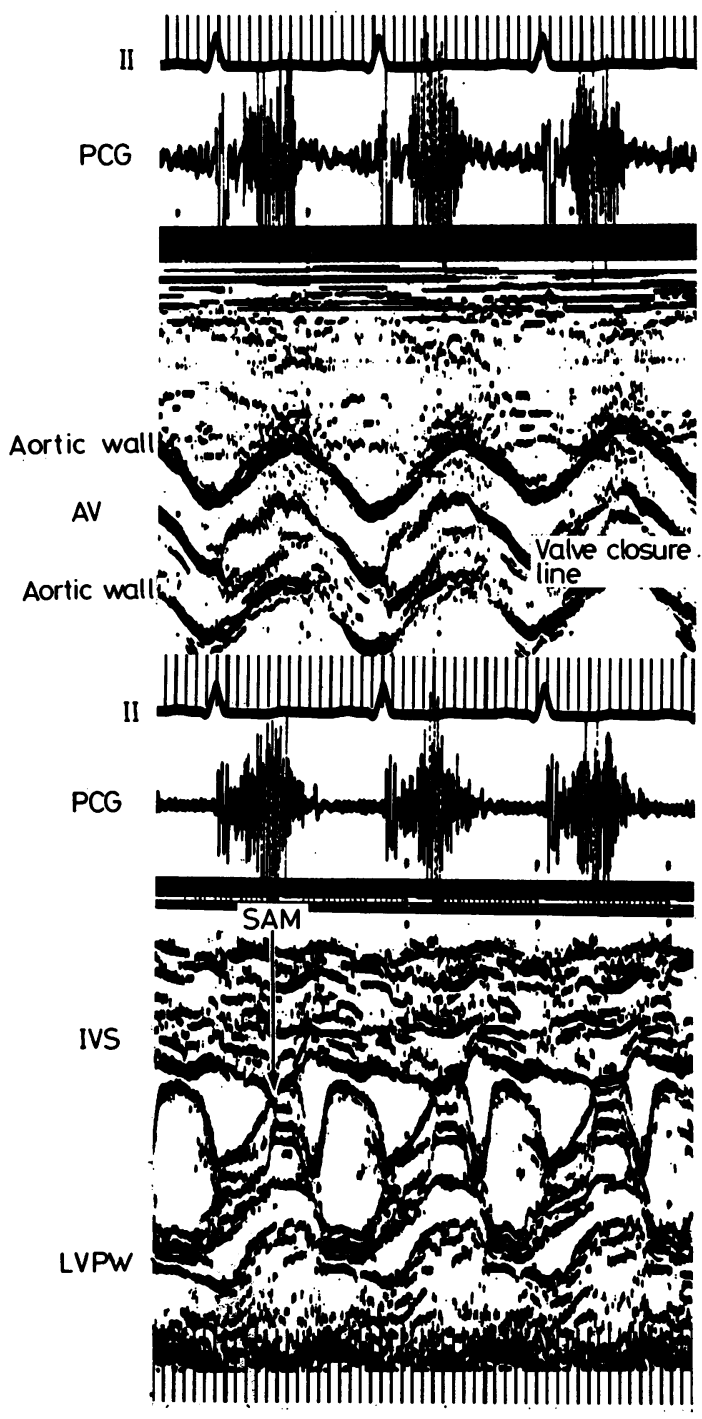

Fig 2 Preoperative echocardiogram showing $S A M$ (arrowed) of the mitral valve. The aortic valve $(A V)$ shows coincident mid and late systolic closure. $I V S=$ interventricular septum, $L V P W=$ left ventricular posterior wall. 
lowed by marked fluttering (fig 1). The mitral valve showed abnormal systolic anterior motion (SAM, arrowed) so that the LVOT appeared obliterated during late systole (fig 2). Coincident with this the aortic valve (AV) opening (approximately $1 \mathrm{~cm}$; normal range $1 \cdot 2-2 \cdot 3 \mathrm{~cm}$ ) was strikingly distorted by partial or almost complete closure. The systolic murmur became maximal as the systolic mitral motion reached its most

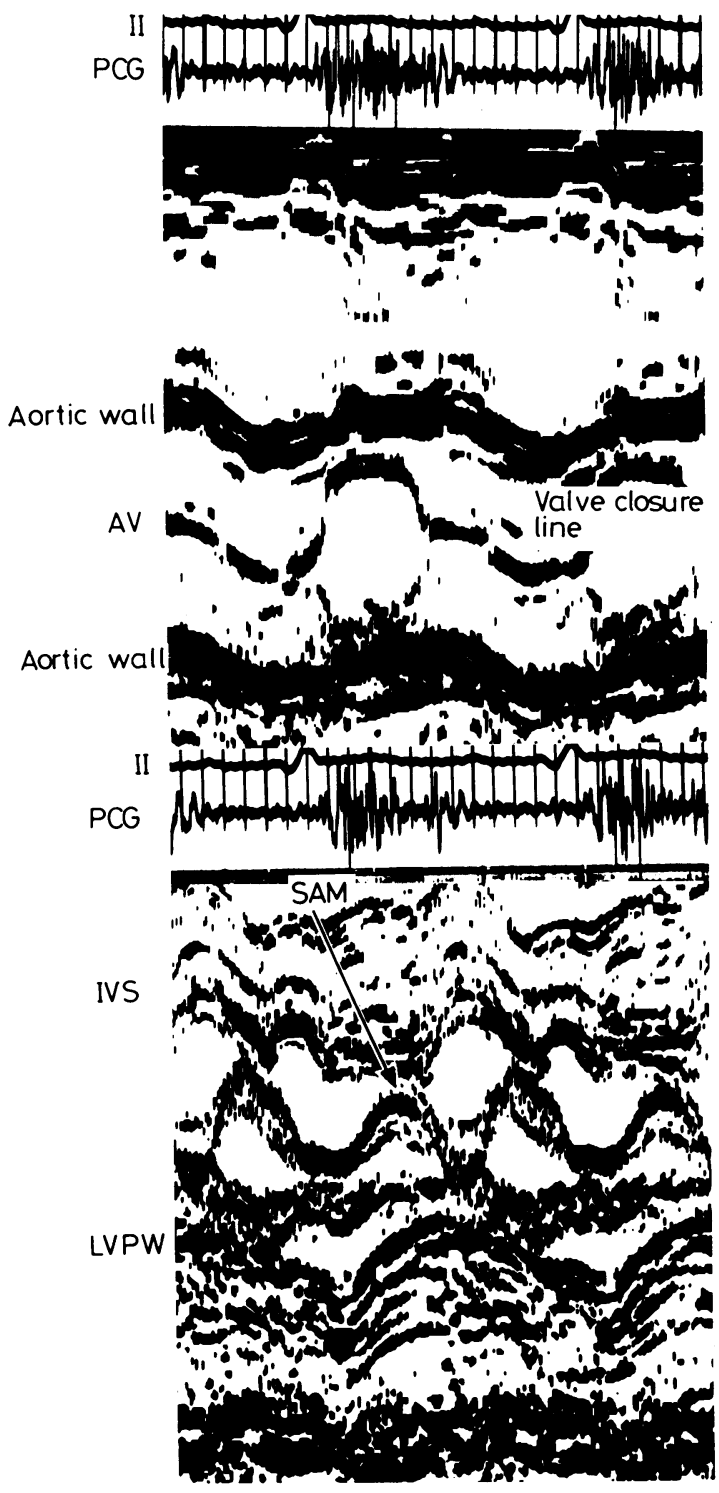

Fig 3 Postoperative echocardiogram showing persistent SAM (arrowed), but normal aortic valve motion. anterior point. The interventricular septum (IVS) and left ventricular posterior wall (LVPW) were thickened (IVS $=1.8 \mathrm{~cm}$ LVPW $=1.5 \mathrm{~cm}$; normal range IVS $=\mathrm{LVPW}=0.7-0.8 \mathrm{~cm} ; \quad \mathrm{IVS} / \mathrm{LVPW}$ ratio $=1 \cdot 2$ ). The left ventricular cavity was small (internal diastolic diameter $3.0 \mathrm{~cm}$; normal range $3 \cdot 3-4 \cdot 5 \mathrm{~cm}$ ).

Echocardiography was repeated 14 days after operation. The aortic valve opening was $1.3 \mathrm{~cm}$, and cusp motion was normal (fig 3), showing neither the early systolic partial closure nor the late systolic deformation of the opening associated with SAM of the mitral valve. Pronounced SAM was still present although the systolic murmur during this phase was reduced in intensity (fig 3).

\section{Discussion}

The echocardiographic signs of DSAS are early aortic partial closure, marked fluttering of the aortic cusps, ${ }^{3}$ and narrowing of the LVOT. ${ }^{4}$ Such findings are specific for DSAS, ${ }^{5}$ and distinguish it from idiopathic hypertrophic subaortic stenosis, which is identified by an adynamic septum, ${ }^{\circ}$ asymmetrical septal hypertrophy, ${ }^{\top}$ and typical mitral systolic anterior motion. " Partial aortic valve closure may also occur but is later in systole than that seen in DSAS. ${ }^{-}$In some patients with DSAS the subaortic membrane may be directly recorded, ${ }^{4}$ but differentiation from SAM of the mitral valve is possible because the membrane is anterior to the mitral valve throughout the cardiac cycle and is clearly separate from the mitral valve in systole and late diastole. ${ }^{9}$ The patient reported here shows the specific echocardiographic features of DSAS but has in addition typical SAM of the mitral valve with associated mid to late systolic partial closure of the aortic valve. Since abnormal SAM of the mitral valve is highly specific for idiopathic hypertrophic subaortic stenosis the presence of coexisting hypertrophic obstruction and fixed subaortic obstruction was diagnosed. This diagnosis is further supported by the pattern of valve motion seen before operation.

The genesis of the features suggesting hypertrophic subaortic stenosis is, however, open to debate. It is possible that DSAS and hypertrophic subaortic stenosis coexist as two separate primary entities, or that the hypertrophic element is compensatory to the afterload imposed by the fixed LVOT obstruction. Furthermore, since the fibromuscular diaphragm was attached to the anterior mitral valve leaflet a further mechanism for interference with the motion of the leaflet can be 
envisaged. Hypertrophic subaortic stenosis has previously been reported to coexist with DSAS, ${ }^{10}$ and echocardiographic evidence for this was presented by others also. ${ }^{11}$ Two patients were described who showed clinical and catheter evidence of hypertrophic subaortic stenosis one to two years after surgical removal of a subvalvar diaphragm. Hypertrophic subaortic stenosis had not been suspected preoperatively, but retrospective examination of the preoperative echocardiogram suggested that SAM may have been overlooked, or thought to be related to the diaphragm. Lack of regression of the hypertrophic obstructive features postoperatively suggested that the condition was not secondary but was a coexistent primary diagnosis. In our patient operative removal of the diaphragm abolished the echocardiographic features of DSAS but SAM persisted. It is notable that the aortic valve no longer showed abnormal closure related to the SAM, but only catheterisation could establish whether this represented abolition of obstruction.

The facts presented have several important implications. Firstly, the results of surgery in type 2 DSAS are not satisfactory, ${ }^{2}$ in that pressure gradients across the LVOT may be unrelieved or increased on subsequent investigation. Evidence of hypertrophic obstruction on the preoperative echocardiogram would warrant detailed invasive study with gradient provocation to determine the relative contribution of the fixed and variable obstructive elements. Secondly, such evidence may allow anticipation of "stone heart" left ventricle with a severe low cardiac output state on cessation of cardiopulmonary bypass as shown by our patient. Under these circumstances the use of inotropic agents such as isoprenaline would be contraindicated since they would tend to increase the obstruction. Thirdly, if the hypertrophic subaortic stenosis is present as a primary entity, the prognosis is related to the natural history of that condition despite successful surgical removal of the sub-aortic diaphragm. We conclude that the diagnosis of DSAS by echocardiography should prompt a careful search for the presence of coexisting hypertrophic LVOT condition.

\section{References}

1 Katz NM, Buckley MJ. Liberthson RR. Discrete membranous sub-aortic stenosis. Report of 31 patients, review of the literature, and delineation of management. Circulation 1977; 56:1034-8.

2 Kelly DT, Wulfsberg BA, Rowe RD. Discrete sub-aortic stenosis. Circulation 1972; 46:309-22.

3 Davis RH, Feigenbaum H, Chang S, Konecke L, Dillon J. Echocardiographic manifestations of discrete sub-aortic stenosis. Am J Cardiol 1974; 33:277-80.

4 Popp RL, Silverman JF, French JW, Stinson EB, Harrison DC. Echocardiographic findings in discrete subvalvular aortic stenosis. Circulation 1974; 49:226-31.

5 Krueger SK, French JW, Forker AD, Caudill CC, Popp RL. Echocardiography in discrete subaortic stenosis. Circulation 1979; 59:506-13.

6 Rossen RM, Goodman D, Ingham RE, Popp RL. Ventricular systolic septal thickening and excursion in idiopathic hypertrophic sub-aortic stenosis. N Engl J Med 1974; 291:1317-9.

7 Henry WL, Clark CE, Epstein SE. Asymmetric septal hypertrophy; echocardiographic identification of the pathognomonic anatomic abnormality of IHSS. Circulation 1973; 47:225-33.

8 Popp RL, Harrison DC. Ultrasound in the diagnosis and evaluation of therapy of idiopathic hypertrophic sub-aortic stenosis. Circulation 1969; 40:905-14.

9 Caudill CC, Krueger SK, Wilson CS, Rourke TR, Policky DB, Weaver WF. Membraneous sub-aortic stenosis complicated by aneurysm of the membraneous septum and mitral valve prolapse. Circulation 1976; 53:580-3.

10 Block PC, Powell WJ, Dinsmore RE, Goldblatt A. Co-existent fixed congenital and idiopathic hypertrophic sub-aortic stenosis. Am J Cardio$1973 ; 31: 523-6$.

11 Chung KJ, Manning JA, Gramiak R. Echocardiography in co-existing hypertrophic subaortic stenosis and fixed left ventricular outflow obstruction. Circulation 1974; 49:673-7. 